\title{
Neutropenia in rheumatoid arthritis: studies on possible contributing factors
}

\author{
ROGER C. BUCKNALL,${ }^{1}$ PAUL DAVIS,${ }^{2}$ PAUL A. BACON,${ }^{1}$ AND \\ JOHN VERRIER JONES ${ }^{3}$ \\ From the ${ }^{1}$ Royal National Hospital for Rheumatic Diseases, Bath; the ${ }^{2}$ Rheumatic Diseases Unit, Department of \\ Medicine, University of Alberta, Edmonton, Canada; and the ${ }^{3}$ Department of Rheumatology, \\ Rush-Presbyterian - St Luke's Medical Center, Chicago, Illinois, USA
}

SUMMARY Twenty-five patients with rheumatoid arthritis (RA) and neutropenia, of whom 19 had a palpable spleen, were compared with 24 patients with uncomplicated RA, and 16 patients with RA and associated splenomegaly without neutropenia. Clinically patients with neutropenia had evidence of a more systemic disease as assessed by prevalence of rheumatoid nodules, weight loss, and recurrent infections. However, there was less evidence of active synovitis than in the other 2 groups. Marrow neutrophil reserve was studied by means of a hydrocortisone stimulation test and was found to be lower in all 3 groups than in normal persons but with most marked depletion in the neutropenic group. Removal of the spleen in some patients with neutropenia resulted in a significant increase in marrow neutrophil reserve. Circulating immune complexes as detected by anticomplementary activity and platelet aggregation tests were detected in $68 \%$ of the RA neutropenia group, $31 \%$ of the RA splenomegaly group, and $8 \%$ of the uncomplicated RA group. Our results show that, assessed both clinically and by the above tests, patients with neutropenia have a greater prevalence of abnormalities which may be directly related to their neutropenia. Patients with splenomegaly alone closely resemble patients with uncomplicated RA.

Neutropenia is an uncommon feature of rheumatoid arthritis. Although it may be seen as an isolated extra-articular manifestation of the disease it is commonly associated with splenomegaly and referred to as Felty's syndrome. The mechanism of neutropenia in RA is unclear but probably multifactorial, reflecting the balance between marrow neutrophil reserves and release, and peripheral neutrophil consumption, destruction, and sequestration. This study was undertaken to assess some of the factors which may influence this balance.

Marrow neutrophil reserves have been tested by a hydrocortisone stimulation test to determine whether reduced reserves may contribute to the leucopenia. The factors resulting in increased destruction are less clear. Previous studies have shown that circulating neutrophils from neutropenic RA patients contain immunofluorescent staining cytoplasmic inclusions which may be the result of ingestion of circulating immune complexes. ${ }^{12}$ This might

Accepted for publication 11 May 1981.

Correspondence to Dr R. C. Bucknall, Link 7C, Royal Liverpool Hospital, Prescot Street, Liverpool L7 8XP. lead to increased splenic sequestration and reduced neutrophil half life. ${ }^{34}$ We have therefore, also assessed the presence of circulating immune complexes in the serum of these patients.

\section{Materials and mathods}

PATIENTS STUDIED

Patients were selected from inpatients in the Bath and Bristol clinical area, which has a population of about 1.25 million. Sixty-five patients with definite or classical RA (ARA criteria ${ }^{5}$ ) and 15 normal controls were studied. All individuals gave their informed consent for investigation.

Twenty-five patients with RA and associated neutropenia were studied and compared with 24 patients with uncomplicated RA. Neutropenia was defined as a neutrophil count of less than $2.0 \times$ $10^{3} / \mathrm{mm}^{3}$. As 19 of our 25 neutropenic patients also had splenomegaly (Felty's syndrome), they were also compared with a second control group consisting of 16 patients with RA and splenomegaly but with persistently normal white counts. Assessment of spleen size was limited to palpation. All patients had long- 
Table 1 Clinical features

\begin{tabular}{llll}
\hline & $\begin{array}{l}\text { Uncomplicated } \\
R A\end{array}$ & $\begin{array}{l}R A / \\
\text { splenomegaly }\end{array}$ & $\begin{array}{l}R A / \\
\text { neutropenia }\end{array}$ \\
\hline No. of pateints & 24 & 16 & 25 \\
Mean age & 55 & 54 & 61 \\
Mean duration of & & & \\
$\quad$ disease (years) & 16 & 9 & 19 \\
Splenomegaly & 0 & 16 & 19 \\
Neutropenia & 0 & 0 & 25 \\
Active synovitis & 24 & 16 & 5 \\
Rheumatoid nodules & 6 & 3 & 13 \\
Weight loss & 3 & 4 & 15 \\
Recurrent infections & 0 & 2 & 15 \\
\hline
\end{tabular}

standing joint disease of duration exceeding 4 years. None of the patients had received, or were on therapy with, gold, D-penicillamine, or cyclophosphamide. One neutropenic patient had previously received azathioprine and another phenylbutazone.

\section{MARROW NEUTROPHIL RESERVES}

The hydrocortisone stimulation test was performed on the 15 normal controls, and 24 patients with uncomplicated RA, 16 patients with RA and splenomegaly, and 24 patients with RA and neutropenia. Nine patients in the RA-neutropenia group were subsequently subjected to splenectomy, and in this group the hydrocortisone stimulation test was repeated 6 months to 2 years postoperatively. The test was carried out with the patient in a resting state at $0900 \mathrm{~h}$. A venous blood sample was collected and the total white cell count measured electronically with a Coulter counter. Differential counts were made on air dried, stained smears by a single technician. Hydrocortisone hemisuccinate $100 \mathrm{mg}$ was administered intravenously. The patient continued to rest, and at the end of 4 hours a further venous blood sample was collected and the total white cell count and differential count measured. The increase in absolute number of neutrophils between 0 and 4 hours was calculated $(\Delta N)$ and the results analysed statistically by means of the $t$ test and Wilcoxon's 2-sample rank test.

\section{TEST FOR COMPLEMENT COMPONENTS AND}

\section{CIRCULATING IMMUNE COMPLEXES}

Levels of the complement component $\mathrm{C} 3$ (normal range $40-88 \mathrm{mg} / \mathrm{dl}$ ) and $\mathrm{C} 4$ (normal range $20-40$ $\mathrm{mg} / \mathrm{dl}$ ) were estimated by the Mancini radial immunodiffusion method. ${ }^{6}$ Breakdown products of C3 (C3i) were detected by crossed immunoelectrophoresis.?

The presence of anticomplementary activity was assessed by means of a haemolytic assay, the indicator system being sheep red cells coated with horse antisheep red cell antibody. ${ }^{8}$ Sera were incubated at both $4^{\circ} \mathrm{C}$ and $37^{\circ} \mathrm{C}$ overnight with guinea-pig complement before adding the sensitised sheep red cells to the system.

The platelet aggregation test was carried out according to the method of Penttinen and Myllyla. ${ }^{9}$ Heat aggregated IgG was used as a control, and to exclude the possibility of aggregation by platelet antibodies the test was performed at both $\mathrm{pH} 7.8$ and $6 \cdot 5$ (aggregation due to antibodies occurs only at this $\mathrm{pH})$. Aggregation by serum at a dilution of greater than 1 in 16 was taken as significant.

\section{ANTINUCLEAR ANTIBODIES}

Immunofluorescent techniques, with human blood buffy coat smears and rat liver sections, were used to detect granulocyte specific and other antinuclear antibodies by means of a standard technique.

Antibodies to single-stranded DNA were measured by a modified Farr technique ${ }^{10}$ and to native DNA by a Millipore filter assay using a synthetic polynucleotide, poly dAT. ${ }^{11}$ Quality control of nuclear antigens was assessed by an ethidiun bromide fluorescence assay. ${ }^{12}$

\section{Results}

CLINICAL FEATURES (Table 1)

The sex distribution and mean ages of all groups were similar. All patients had synovitis of at least 4 years' duration. But the mean duration of disease in the patients with uncomplicated rheumatoid arthritis and RA with neutropenia was significantly longer than in the patients with RA and splenomegaly alone. By selection no patients in the uncomplicated RA group had splenomegaly, while this was a feature in all 16 patients in the RA and splenomegaly group. Splenomegaly was also found in 19 of the 25 patients with RA and neutropenia and thus would fulfil the clinical criteria for the diagnosis of Felty's syndrome. Neutropenia by selection was not a feature of any of the patients in the uncomplicated RA or RA splenomegaly group but was present in all patients in the RA neutropenia group. Major clinical differences were seen in the 3 groups. The most striking difference was that both sets of patients in the uncomplicated RA and RA splenomegaly group had evidence of active synovitis at the time of entry to the study. However, this was a feature in only 5 of the $25(20 \%)$ of the patients with RA and neutropenia, who despite prolonged disease duration, and in many cases extensive joint deformity, had less active synovitis. Nodules were also more frequent in the patients with neutropenia, occurring in 13 of $25(52 \%)$ but only in approximately $25 \%$ of the other 2 groups. Weight loss and other general systemic disturbances were also a feature of the RA neutropenic patients and 
were less common in the other 2 groups. Another major difference in the patient groups was the presence of recurrent infections. These were found almost entirely in the patients with neutropenia, occurring in 15 of the $25(60 \%)$. No recurrent infections were seen in the patients with uncomplicated RA and in only 2 of the 16 patients with RA and splenomegaly.

Antinuclear antibodies (Table 2) were present in the sera of 21 of the 25 RA-neutropenia patients and in a smaller proportion of patients in the 2 control RA groups. This finding was nonspecific, the antibodies reacting both with rat liver cell as well as with human granulocyte nuclei. Antibodies to ss-DNA were detected by the Farr assay in the sera of all patients with RA-neutropenia, all patients with RA and splenomegaly, but in none of 9 patients with uncomplicated RA. No sera from any group contained antibodies to poly-dAT.

Thus major differences were seen in the 3 groups studied, with patients with RA-neutropenia having

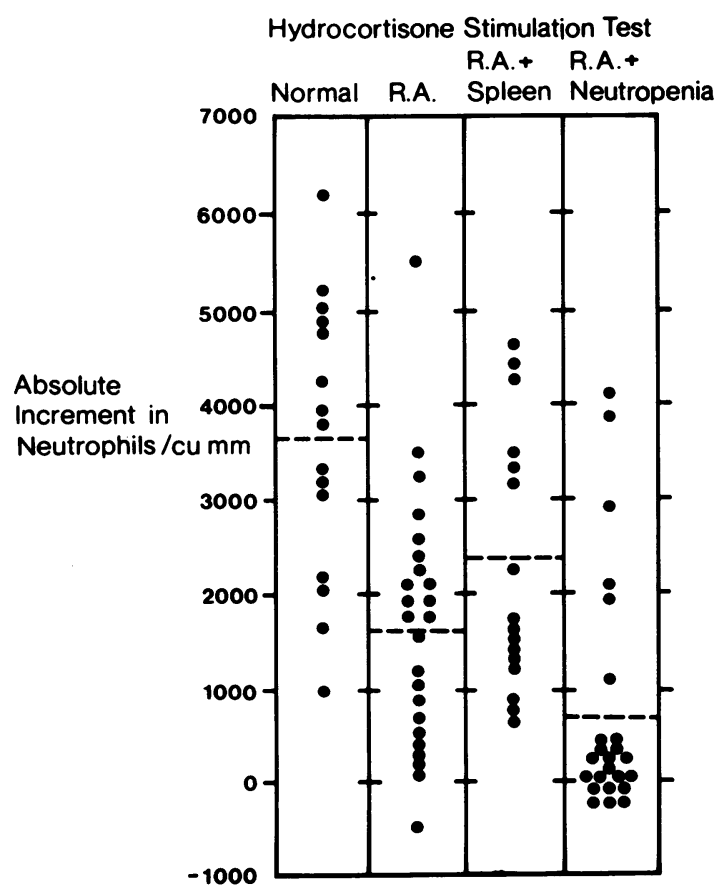

Fig. 1. Marrow neutrophil reserve as assessed by the hydrocortisone stimulation test. In all 3 groups of rheumatoid patients there was a significant depression of marrow neutrophil reserve compared with the normal controls (RA and RA/neutropenia $p<0.001 ; R A$ splenomegaly $p<0.01)$. There was a significant difference between the RA/neutropenia and other $2 R A$ groups ( $R A$ alone $0.01<p<0.5 ; R A /$ splenomegaly $p<0.001$ ).
Table 2 Antinuclear and DNA antibodies

\begin{tabular}{llcl}
\hline & $\begin{array}{l}\text { Uncomplicated } \\
R A\end{array}$ & $\begin{array}{l}R A / \\
\text { splenomegaly }\end{array}$ & $\begin{array}{l}\text { RA/ } \\
\text { neutropenia }\end{array}$ \\
\hline ANA & $5 / 10$ & $7 / 16$ & $21 / 25$ \\
Ab s-s DNA & $0 / 9$ & $16 / 16$ & $25 / 25$ \\
Ab poly-dAT & $0 / 9$ & $0 / 14$ & $0 / 12$ \\
\hline
\end{tabular}

Antinuclear antibodies were detected in all 3 groups. Antibodies to single stranded DNA were restricted to the splenomegaly and neutropenia groups, but antibodies to synthetic native DNA, with poly-dAT used as antigen, were not detected in any group.

less evidence of active synovitis but more evidence of severe disease and its complications as shown by the evidence of nodules, antinuclear antibodies, weight loss, and recurrent infections. These changes were particularly significant when comparison was made with the patients with uncomplicated RA. The patients with splenomegaly but with persistently normal white cell counts were clinically similar to the patients with uncomplicated RA, suggesting that the presence of splenomegaly alone is not of serious clinical import unless associated with neutropenia as in our RA-neutropenic patients.

\section{HYDROCORTISONE STIMULATION TEST}

The results are shown in Fig. 1 . There was a highly significant difference $(p<0 \cdot 001)$ between the mean response in the normal control group $(\Delta N=$ $\left.3746 / \mathrm{mm}^{3}\right)$ and the uncomplicated RA group $(\Delta \mathrm{N}=$ $\left.1743 / \mathrm{mm}^{3}\right)$. The patients with RA and splenomegaly showed a mean response of $2295 / \mathrm{mm}^{3}$, a significant difference from the normal controls $(p<0.01)$, but no difference from the uncomplicated RA group $(\mathrm{p}>0 \cdot 10)$.

The mean response in the RA neutropenia group was $749 / \mathrm{mm}^{3}$, showing a highly significant difference

Table 3 Complement levels and circulating immune complexes

\begin{tabular}{llll}
\hline & $\begin{array}{l}\text { Uncomplicated } \\
R A\end{array}$ & $\begin{array}{l}\text { RA/ } \\
\text { splenomegaly }\end{array}$ & $\begin{array}{l}\text { RA/ } \\
\text { neutropenia }\end{array}$ \\
\hline No. of patients & 12 & 16 & 25 \\
Depressed C3 & 0 & 0 & 6 \\
$\begin{array}{c}\text { Depressed C4 } \\
\begin{array}{c}\text { Immune complexes } \\
\text { Anticomplementary } \\
\text { activity }\end{array}\end{array}$ & 0 & 4 & 7 \\
$\begin{array}{c}\text { Platelet aggregation } \\
\text { Total (one of both tests } \\
\text { positive) }\end{array}$ & 1 & & \\
\hline
\end{tabular}

Serum C3 levels were depressed in 6 of the neutropenic patients but in none of the other groups.

Circulating immune complexes were detected by one or other test (AC or Pl Agg) in 17 of 25 neutropenic patients, 5 of 16 with RA and splenomegaly, and only 1 of 12 uncomplicated RA patients. (Uncomplicated RA/RA

splenomegaly, $x^{2}<1 ; p>0 \cdot 10$, NS. Uncomplicated RA/RA neutropenia, $x^{2}$ 9.29; $<<0.01$. RA/splenomegaly/RA neutropenia $\chi^{2} 3.92 ; 0.01<p<0.05$.) 
Hydrocortisone Stimulation Test Splenectomy

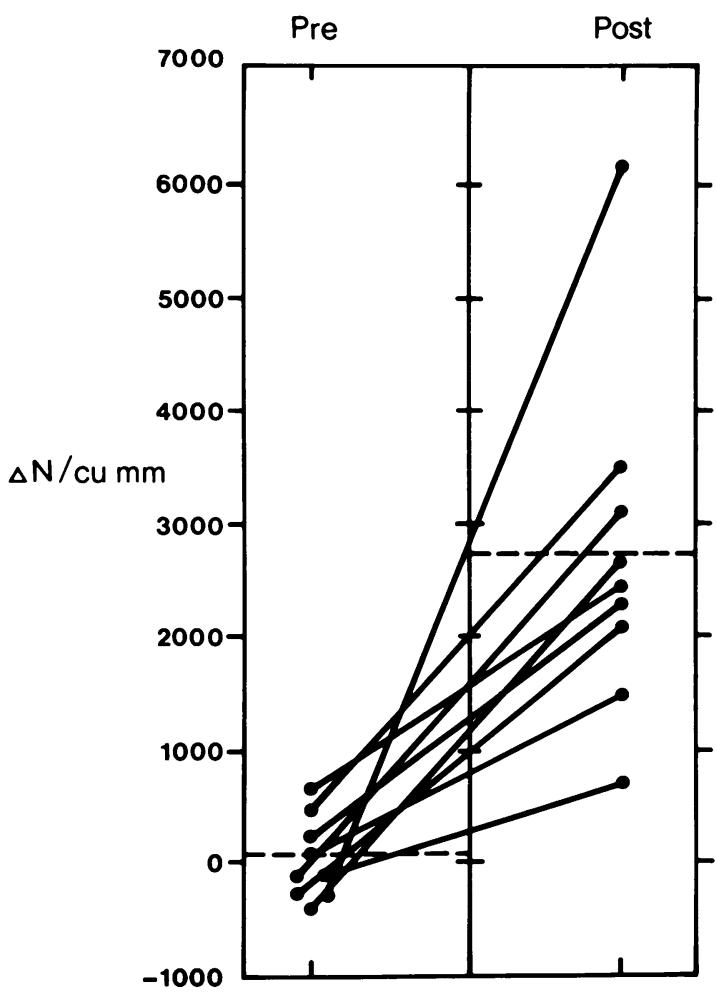

Fig. 2 Marrow neutrophil reserve as assessed by the hydrocortisone stimulation test before and after splenectomy. All patients tested showed an increase in marrow reserve with a mean absolute increase in neutrophils $(\Delta N)$ in 9 patients before splenectomy of $87 / \mathrm{mm}^{3}$ and after splenectomy $2737 / \mathrm{mm}^{3}(p<0 \cdot 001)$.

from both the normal controls and the RAsplenomegaly group $(0 \cdot 01<p<0 \cdot 05)$. Analysis of the results in the RA-neutropenia group showed that there was no significant difference between those with splenomegaly $\left(\Delta \mathrm{N}=732 / \mathrm{mm}^{3}\right)$ and without splenomegaly $\left(797 / \mathrm{mm}^{3}\right)$. Patients with RA and splenomegaly had results similar to those of the uncomplicated RA patients and significantly different from those of the RA neutropenia patients irrespective of the presence of splenomegaly.

The results of the hydrocortisone test performed before and after splenectomy in 9 RA neutropenia patients are shown in Fig. 2. All patients showed an increased response to hydrocortisone. The mean response before splenectomy was $87 / \mathrm{mm}^{3}$ compared with $2737 / \mathrm{mm}^{3}$ after the operation $(p<0 \cdot 001)$.
TESTS FOR COMPLEMENT COMPONENTS AND CIRCULATING IMMUNE COMPLEXES (Table 3 ) C3 levels were normal or elevated in the majority of patients in all 3 rheumatoid groups. However, depressed C3 levels occurred in 6 of the RA-neutropenia groups but in none of the control groups. Depressed levels of $\mathrm{C} 4$ were seen in both the RA splenomegaly and RA-neutropenia groups but not in those patients with uncomplicated RA. Evidence of complement activation was detected in 3 of 6 RA-neutropenia patients tested, there being $50 \%$ activation in 2 patients and $100 \%$ in one patient. Complement activation was not detected in any patients in the 2 control groups.

Anticomplementary activity was detected in the sera of 13 of 25 RA-neutropenia patients and in 2 of these in very high titre $(>4096)$. It was present in only 2 of the 16 RA-splenomegaly group but again in very high titre (4096) and in only 1 of the 12 uncomplicated RA group. The platelet aggregation test was positive in 10 of $18 \mathrm{RA}$-neutropenia patients studied but also in 4 of the $11 \mathrm{RA}$-splenomegaly group and 1 of the uncomplicated RA group. Circulating immune complexes were detected by one or more of the 3 methods in a total of 17 of 25 RA-neutropenia sera compared with 1 of 12 uncomplicated RA sera $\left(\chi^{2}=\right.$ $9 \cdot 29 ; \mathrm{p}<0.01)$, and 5 of the 16 RA-splenomegaly sera $\left(\chi^{2}=3.92 ; 0.01<\mathrm{p}<0.05\right)$.

There was no correlation between the degree of neutropenia, or the degree of impairment of marrow neutrophil reserve, and the tires of circulating immune complexes.

\section{Discussion}

This study was undertaken to assess factors which may influence the development of neutropenia in patients with rheumatoid arthritis. Studies on marrow neutrophil reserve and circulating immune complexes have been performed on 25 patients with rheumatoid arthritis and neutropenia and compared with those on 24 patients with uncomplicated RA. Nineteen of our 25 patients with neutropenia also had splenomegaly and would fulfil accepted clinical criteria for the diagnosis of Felty's syndrome. An additional control group of individuals with rheumatoid arthritis and splenomegaly but without neutropenia have therefore also been studied. Clinically major differences were seen in the groups. As expected, patients with RA and neutropenia had clinical evidence of more severe disease as shown by the presence of rheumatoid nodules, antinuclear antibodies, weight loss, and recurrent infections. Although the mean duration of disease was similar in the groups, an interesting observation was the relative lack of synovitis in the patients with RA and 
neutropenia when compared with the uncomplicated rheumatoid patients. Perhaps more surprising were the findings in patients with RA and splenomegaly alone. These patients were clinically more similar to the uncomplicated RA patients than the patients with RA and neutropenia. In particular, although they had a slightly shorter duration of disease, a similar prevalence of active synovitis, rheumatoid nodules, and weight loss were noted when compared to the uncomplicated group. Recurrent infections, which were so prevalent in the patients with RA and neutropenia, were not a feature of their disease. Since the clinical association between neutropenia, polyarthritis, and splenomegaly as described by Felty ${ }^{13}$ was not a feature of all our patients with neutropenia, it would appear that the presence of neutropenia was of more clinical significance than the presence of splenomegaly. This observation supports the findings in cases described by Spivak, ${ }^{14}$ who in reviewing patients with neutropenia concluded that a palpable spleen was not an obligatory feature of Felty's syndrome.

Laboratory studies in the 3 groups of patients also showed differences that support our clinical findings. In general the patients with RA and splenomegaly behaved in a similar way to patients with uncomplicated RA. Major differences were seen in these 2 groups when compared with the patients with RA and neutropenia whether or not a spleen was palpable. We have shown by the hydrocortisone stimulation test a marked depletion of marrow neutrophil reserve in patients with RA and neutropenia, although abnormal results were also seen in some patients without neutropenia. Marrow neutrophil reserve has been assessed by various methods, including pyrogens ${ }^{15}$ and aetiocholanolone, ${ }^{16}$ and with the latter an impaired response has been found in RA with neutropenia. In our study we have used intravenous hydrocortisone and have found it to be a simple technique which can be easily applied to a large number of patients. Dale et al. ${ }^{17}$ have recently compared this technique with pyrogens and aetiocholanolone. They concluded that hydrocortisone has 2 advantages over the other methods, namely, that it is free of side effects and produces a smaller degree of variation in response in normal subjects. The origin of neutrophils detected in the circulation after hydrocortisone administration has not been established in this study, but Bishop et al. ${ }^{18}$ using $\mathrm{DF}^{32} \mathrm{P}$-labelled neutrophils showed that they arose from the marrow and not from the marginated pool.

The mechanism of marrow depression in the neutropenia of rheumatoid arthritis may relate to the influence of the spleen as shown by the marked improvement in neutrophil release in response to hydrocortisone in the 9 patients studied after splenectomy. Deubelbeiss and Roth ${ }^{19}$ have shown an impaired response to hydrocortisone in patients with splenomegaly due to various pathological processes and also significantly higher responses were seen in splenectomised patients compared with those with a normal sized spleen. Kimball et al. ${ }^{16}$ proposed that the spleen produces a humoral marrow inhibiting factor since the reinfusion of plasma removed after splenectomy had a mild inhibitory effect on marrow neutrophil release in response to aetiocholanolone. The reinfusion of plasma removed postoperatively did not have this effect. We have previously shown that when serum from these patients is added to an in-vitro culture of normal granulocytes there is an inhibition of normal granulocyte development. ${ }^{20} \mathrm{Our}$ results would support the hypothesis that decreased marrow neutrophil reserves is an important factor in the neutropenia observed in our patients, but that this is reversible after splenectomy.

What leads to this decreased marrow reserve is less clear, but it could be the end result of ingestion by phagocytes of circulating immune complexes and splenic sequestration. ${ }^{21}$ Using 2 techniques, we have shown that the serum of patients with RA and neutropenia contain high levels of circulating immune complexes with a greater prevalence than the patients with uncomplicated RA and RA with splenomegaly, these differences being statistically significant. These observations are in accordance with those recently published by Hurd et al. ${ }^{22}$ who, using Clq binding assay, showed that $60 \%$ of patients with Felty's syndrome had circulating immune complexes compared with $10 \%$ of patients with uncomplicated RA. These results would suggest that circulating immune complexes may be an important contributing factor in patients with neutropenia and RA. However, in our study no differences were seen between the patients with RA, neutropenia and splenomegaly, and RA with neutropenia alone, suggesting that the correlation is stronger between circulating immune complexes and neutropenia than with circulating immune complexes and splenomegaly.

In conclusion we have shown that the neutropenia of rheumatoid disease may relate either to the presence of circulating immune complexes or to a reduced marrow neutrophil reserve, perhaps due to a splenic inhibiting factor. It is not clear, however, whether these variables are interrelated. On the basis of the results of this study we would suggest that in terms of the pathogenesis of the neutropenia such patients should be considered as 2 separate groups. In this way a rational approach to therapy with treatment directed towards either a circulating immune complex disease or splenectomy may be considered. 
We thank the physicians in the Bath and Bristol clinical areas who allowed us to study patients under their care. We are also grateful to R. H. Cumming, PhD, and P. Panayiotou, who performed the immune complex tests, Mrs J. Crokett, who carried out the hydrocortisone stimulation tests, Mrs E. Collins for statistical analysis, and Miss J. Savage for secretarial help.

\section{References}

${ }^{1}$ Hurd E R, Andreis M, Ziff M. Phagocytosis of immune complexes by polymorphonuclear leucocytes in patients with Felty's syndrome. Clin Exp Immunol 1977; 28: 413-25.

${ }^{2}$ Hurd E R, Lospalluto J, Ziff M. The role of immune complexes in production of the neutropenia of Felty's syndrome (abstract). $J$ Rheumatol 1974; 1 (suppl): 105.

${ }^{3}$ Bishop C R, Rothstein G, Ashenbrucker H E, Athens J W. XIV Blood neutrophil kinetics in chronic steady state neutropenia. $J$ Clin Invest 1971; 50: 1678-89.

4 Brubaker L H, Johnson C A, Morris A. Evaluation of neutropenia in rheumatic diseases, a simple new approach. VI Pan American Congress in Rheumatology 1974; abs. 193.

s Ropes M W, Bennett G A, Cobb S, Jacox R, Jessar R A. Revision of diagnostic criteria in rheumatoid arthritis. Ann Rheum Dis 1959; 18: 49-53.

- Mancini G, Carbonara A O, Heremans J F L. Immunochemical quantitation of antigens by single radial immunodiffusion. Immunochemistry 1965; 2: 235-54.

7 Clarke H G, Freeman T. Quantitative immunoelectrophoresis of human serum proteins. Clin Sci 1968; 35: 403-13.

8 Johnson A H, Mowbray J F, Porter M A. Detection of circulating immune complexes in pathological human sera. Lancet 1975; i: 762-5.

- Penttinen K, Myllyla G. Interaction in human blood platelets, viruses and antibodies. I. Platelet aggregation test with microequipment. Ann Med Exp Fenn 1968; 46: 188-92.

10 Farr R S. A quantitative immunochemical measure of the primary interaction between radioiodinated BSA and antibody. $J$ Infect Dis 1958; 103: 239-62.
11 Lentz K, Winfield J, Barland P. Antibodies to dAT detected by membrane filtration. Arthritis Rheum 1976; 19: 867-73.

12 Davis P, Burrington M, Russell A S, Morgan A R. Analysis of DNA structure by hydroxypatite columns and ethidium bromide fluorescence techniques. Arthritis Rheum 1978; 21: 407-13.

${ }^{13}$ Felty A R. Chronic arthritis in the adult, associated with splenomegaly and leucopenia. Johns Hopkins Med J 1924; 35: 16-20.

${ }^{14}$ Spivak J L. Felty's syndrome: an analytical review. Johns Hopkins Med J 1977; 141: 156-62.

is Craddock C G, Perry S, Lutz E V, Lawrence J S. Evaluation of marrow granulocytic reserves in normal and disease states. Blood 1960; 15: 840-55.

16 Kimball H R, Wolff S M, Talal N. Plotz P G, Decker J L. Marrow granulocyte reserves in the rheumatic diseases. Arthritis Rheum 1973; 16: 345-52.

${ }^{17}$ Dale D C, Fauci A S, Guerry D, Wolf S M. Comparison of agents producing a neutophilic leucocytosis in man.J Clin Invest 1975; 56: 808-13.

${ }^{18}$ Bishop C R, Athens J W, Boggs D R, Warner H R, Cartwright G E, Wintrobe M M. Leukokinetic studies XIII. A non-steady state kinetic evaluation of the mechanism of cortisone-induced granulocytosis. J Clin Invest 1968; 47: 249-60.

19 Deubelbeiss K A, Roth P. Postmitotic marrow neutrophils and neutrophil mobilization in man: role of the spleen. Blood 1978; 52: 1021-32.

20 Goldberg L S, Bacon P A, Bucknall R C, Fitcher J, Cline M J. Inhibition of human bone marrow granulocyte precursors by serum from patients with Felty's syndrome.J Rheumatol 1980; 7: 275-8.

${ }^{21}$ Hurd E R. Presence of leucocyte inclusions in spleen and bone marrow of patients with Felty's syndrome. J Rheumatol 1978; 5: 26-32.

${ }^{22}$ Hurd E R, Chubick A, Jasin H E, Ziff M. Increased Clq binding immune complexes in Felty's syndrome. Arthritis Rheum 1979; 22: 697-702. 